\title{
Silicon nanophotonic ring resonator sensors integrated in reaction tubes
}

\author{
C. Lerma Arce ${ }^{a-c^{*}}$, A. Goes ${ }^{\text {b-c }}$, E. Hallynck ${ }^{a-c}$, P. Dubruel ${ }^{b-c}$, K. Komorowska ${ }^{a-c}$, S. Van Put ${ }^{d}$, \\ P. Bienstman ${ }^{\mathrm{a}-\mathrm{c}}$ \\ ${ }^{a}$ Photonics Research Group (INTEC) Gent University, Sint-Pietersnieuwstraat 41, 9000 Gent, \\ Belgium; ${ }^{\text {b }}$ Polymer Chemistry and Biomaterials Group, Gent University, Krijgslaan 281 (S4), 9000 \\ Gent, Belgium; ${ }^{\mathrm{c}}$ Center for Nano- and Biophotonics, Gent University, 9000 Gent, Belgium, ${ }^{\mathrm{d}} \mathrm{Center}$ \\ for MicroSystems Technology, Gent University, Technologiepark 914, 9052 Zwijnaarde (Gent), \\ Belgium
}

\begin{abstract}
Enzyme-linked immunosorbent assays (ELISA) are the most popular immunoassay techniques performed every day in hospitals and laboratories and they are used as a diagnostic tool in medicine and plant pathology, as well as a qualitycontrol check in various industries. However, complex labeling techniques are required to be able to perform the assay and non-specific binding and endpoint timing are difficult to optimize. These issues could be solved by label-free techniques such as silicon nanophotonic microring resonator sensors, but this platform requires complex microfluidics, which is very much removed from the daily practice in e.g. hospital labs, which still relies to a large degree on platforms like 96-well microtiter plates or reaction tubes. To address these issues, here, we propose the combination of a simple and compatible reaction tube platform with label free silicon-on-insulator (SOI) photonic biosensors, where the flow is through the sensor chip as opposed to over the chip as in more conventional approaches. This device allows real time detection and analysis. Its great flexibility and small footprint make it ideal for an easy handling in any laboratory.
\end{abstract}

Keywords: Silicon nanophotonic sensors, photonic microring resonators, Silicon-on-Insulator (SOI), reaction tubes, Enzyme-linked immunosorbert assay (ELISA)

\section{INTRODUCTION}

Label-free techniques such as silicon photonic microring resonator sensors attempt to overcome the stability and reliability problems of biosensors relying on the detection of labeled molecules. While labeled detection methods can be sensitive down to a single molecule, labels can structurally and functionally alter the assay and the labeling process is labor intensive and costly. Quantification is difficult since the bias label intensity level is dependent on all working conditions. Moreover a labeled assay can only be performed in an 'end-point' fashion so that no kinetic information on the biomolecular interaction can be obtained. In practice, label-based assays require a high degree of development to assure that the label does not block an important active site on the tagged molecule or modify the molecular conformation.

Due to these considerations, there has been a drive to reduce assay cost and complexity while providing more quantitative information with high throughput. Label-free detection is a solution to this and involves a transducer that directly measures some physical property of the biological compound. The type of biosensor handled in this work is an affinity-based biosensor: a so-called 'receptor' or 'ligand' is attached to the surface of the sensor, which responds to the affinity interaction of the receptor with an analyte. The receptor molecule can be an antibody, receptor protein or DNA. The formation of complexes can thus be monitored continuously and many interactions can be followed simultaneously. This real-time data results in information on the kinetics of the reaction as well as on the concentration of the antibodies in the sample.

Optical label-free biosensors have received considerable attention over the past years. The key behind optical biosensors' ability to detect biological analytes is that they are able to translate changes in the propagation speed of light into a

*cristina.lermaarce@ @intec.ugent.be; phone +32 (0) 9264 3316; fax +32 (0) 9264 3593; 
quantifiable signal proportional to the amount of biological material present on the sensor surface.

Label-free biosensing with silicon nanophotonic microring resonator sensors has proven to be an excellent sensing technique for achieving high-throughput and high sensitivity, comparing favorably with other labeled and label-free sensing techniques. However, as in any biosensing platform, silicon nanophotonic microring resonator sensors require a fluidic component which allows the continuous delivery of the sample to the sensor surface. This is the big disadvantage of this platform since this type of microfluidic system is very much removed from the daily practice in e.g. hospital labs, which still relies to a large degree on platforms like 96-well microtiter plates, or reaction tubes.

Here we propose the combination of a simple and lab-compatible reaction tube platform, with label-free nanophotonic biosensors with a special microfluidic system imbedded in the same chip, where the flow is through the chip as opposed to over the chip as in more traditional approaches. This shows that label-free nanophotonic ring resonators can be also used in the user-friendly platform like reaction tubes or well microtiter plates, conserving their excellent performance.

\section{INTEGRATION: NANOPHOTONIC CHIP, MICROFLUIDIC SYSTEM AND REACTION TUBES.}

\subsection{Nanophotonic ring resonator sensors, chip layout, and setup}

The photonic chip was fabricated in SOI with $2-\mu \mathrm{m}$ buried oxide and a $220-\mathrm{nm}$ silicon top layer with CMOS compatible 193-nm optical lithography and dry etching ${ }^{1}$. The resonators consist of 450-nm-wide single-mode waveguides, with 5$\mu \mathrm{m}$ bend radius, $2-\mu \mathrm{m}$-long directional couplers, and a gap of $180 \mathrm{~nm}$ between the waveguides. The layout of the chip is illustrated in Figure. $1^{2}$ Four rings are connected to one common input waveguide, each of them having a dedicated drop signal port. Three of these four ring series are placed independently next to the other. The three input waveguides are simultaneously addressed through vertical grating couplers ${ }^{3}$ with a 2 -mm-wide collimated beam from a tunable laser source. The output signals of the ring resonators are near-vertically coupled to free space by means of integrated grating couplers and are imaged with an infrared camera.

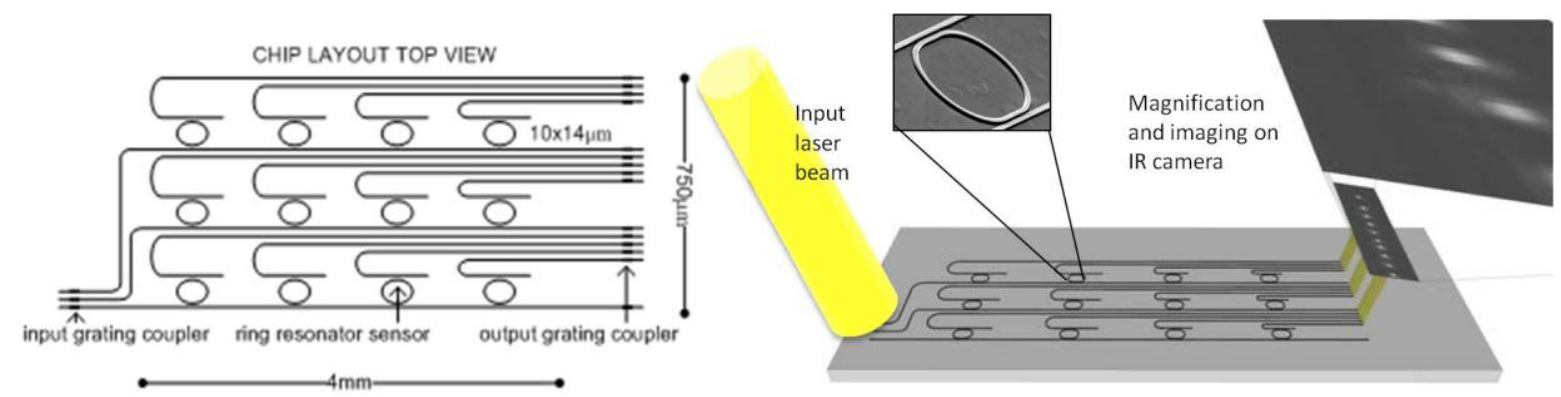

Figure 1. Left. Chip layout top view. Four rings are connected to one common input waveguide, each of them having a dedicated drop signal port. Three of these four ring series are placed independently next to the other. Right: Setup: A SANTEC TSL-510 tunable laser was used as a light source. The three input waveguides are simultaneously addressed through vertical grating couplers with a $2 \mathrm{~mm}$-wide collimated beam from a tunable laser source. The output signals of the ring resonators are near-vertically coupled to free space by means of integrated grating couplers and are imaged with an infrared camera. ${ }^{4}$

The shallow-etched gratings are part of the chip design and have a maximum coupling efficiency of $31 \%$ at a wavelength of $1.55 \mu \mathrm{m}\left(40-\mathrm{nm} 1-\mathrm{dB}\right.$ bandwidth) for a $10^{\circ}$ off-vertical coupling angle. Because the bandwidth of the grating couplers is larger than the free spectral range of the resonators, the grating couplers do not limit the number of resonators placed in series. This optical setup allows very high alignment tolerances, measures the spectrum of all the ring resonators in parallel, and therefore presents no limitation for high-throughput sensing.

A TSL-510 tunable laser was used as a light source. The transmitted light was detected by an infrared camera. The input power was chosen so that the intensity of the resonance peaks corresponds to the pixel saturation level to obtain a maximum signal-to-noise ratio. We have developed software that captures an image for every wavelength step and stores the maximum intensity values within each dedicated area that overlaps with an output grating coupler spot. Postprocessing consists of fitting the spectra to their theoretical shape and tracking these resonance peaks over time. 


\subsection{Embedded microfluidic system.}

We propose a microfluidic system embedded in the photonic chip that will be integrated at the bottom of the reaction tube. This microfluidic system consists of certain apertures that perforate the chip from the top to the bottom. The solution inserted in the tube will flow through these openings that work as exit channels, creating a flow, which will accelerate the detection process.

Figure 2 shows a schematic of the device with the embedded microfluidic system.

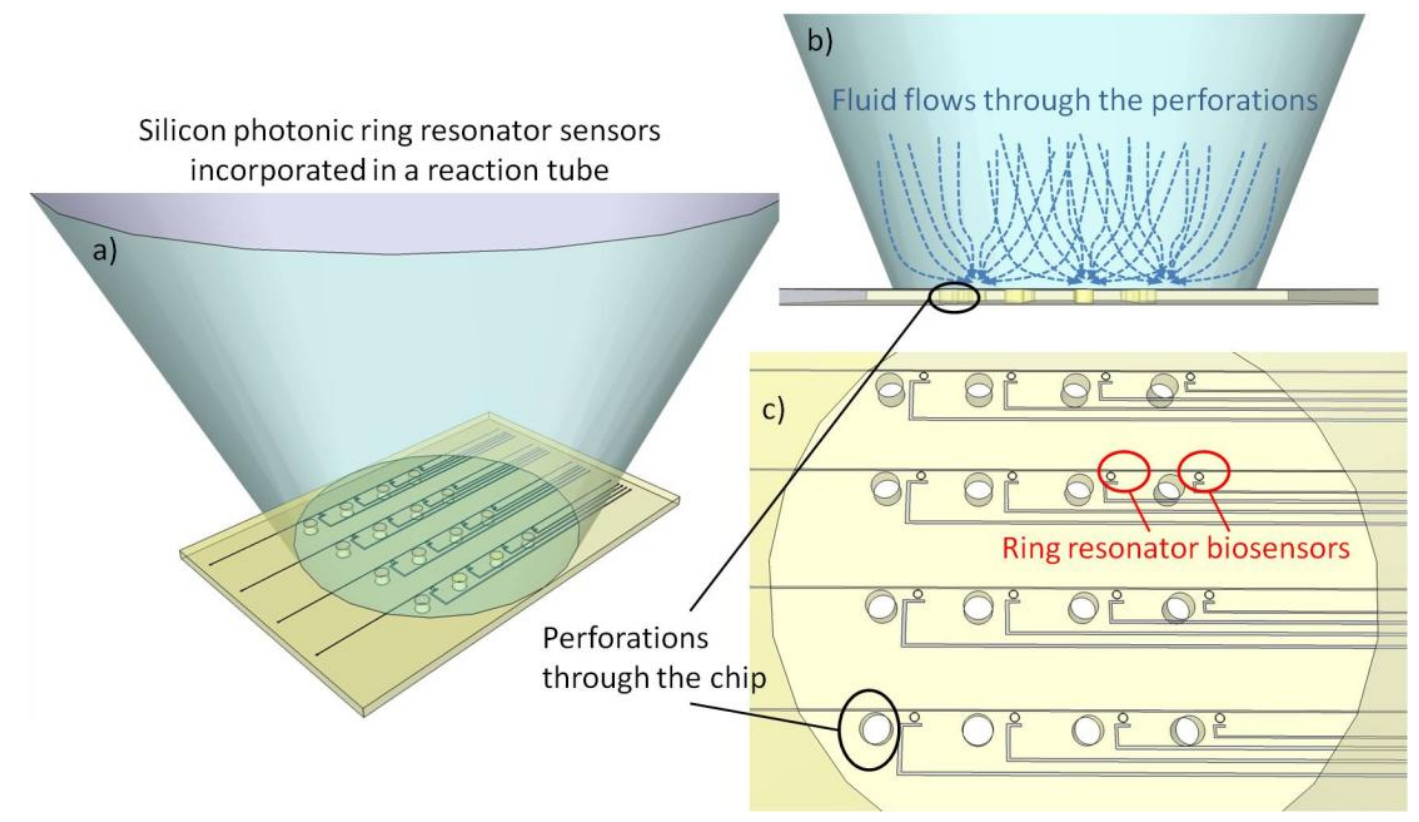

Figure 2. a) Schematic of the device. The silicon-on-insulator chip with the photonic biosensors and the embedded microfluidic system is incorporated at the bottom of a reaction tube. b) The solution inserted in the tube will flow through these openings that work as exit channels, creating a flow, which will accelerate the detection process. c) Layout of the chip. The array of ring resonators sensors is accompanied by an array of perforations next to them.

The perforations of the silicon-on-insulator chip are achieved by means of laser ablation. A Duetto laser source (TimeBandwidth) was used to perform the perforations. 1000 ps-duration pulses were applied with a repetition rate of $50 \mathrm{kHz}$ at $355 \mathrm{~nm}$. The size of the openings and their position can be easily optimized by editing some parameters in the laser.

Some preliminary simulations were performed in order to confirm the existence of flow through the surface of the sensors. Figure 3 shows the streamlines of a water-based fluid that goes through holes of $40 \mu \mathrm{m}$ diameter which simulate the perforations of the chip.

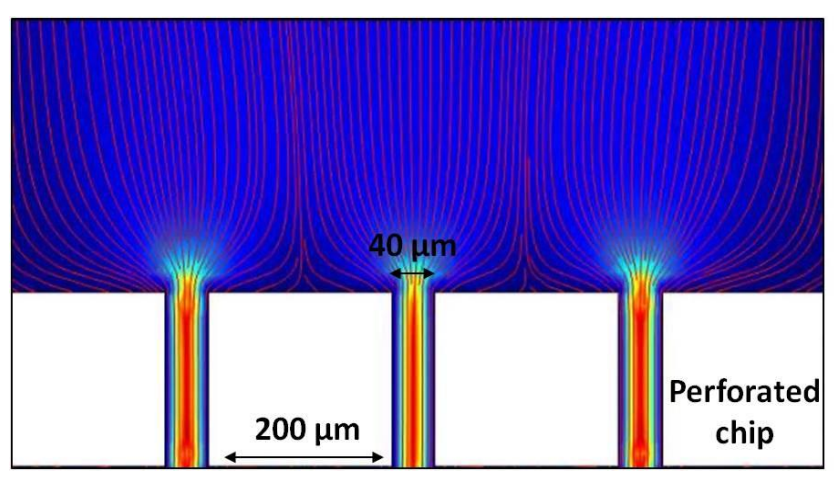

Figure 3. Simulation of a water-based fluid flow in our device structure. Streamlines in red confirm us the existence of a flow in the vicinity of the apertures. 
The position of the perforations must be in the near vicinity of the sensors to warrantee enough flow on their surface. Figure 4 shows the perforations made in the chip. The array of sensors is accompanied by an array of perforations. Each perforation will create a flow for its closest sensor.

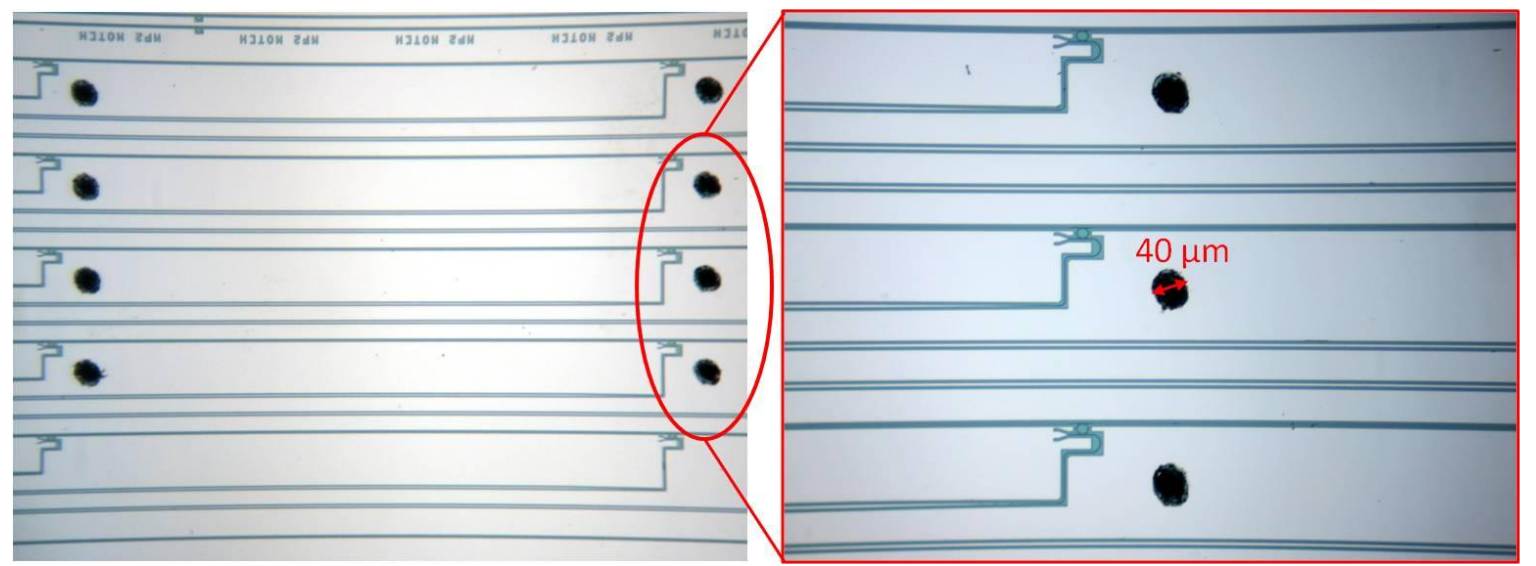

Figure 4. Microscope picture of the array of photonic sensors accompanied by an array of perforations next to them. Each perforation will create a flow in its closest sensor.

\subsection{Integration}

The photonic chip with the array of sensors and the embedded microfluidic system described above is incorporated to the bottom of the reaction tube, once its original bottom is mechanically removed.

The attachment of the chip to the bottom of the tube is done permanently using UV curable glue which allows us to align precisely the array of sensors in the center of the reaction tube. When the fluid under analysis will be inserted in the tube, this will be in contact with the sensors, and flow out through the apertures described above. Figure 5 shows the picture of the final device.

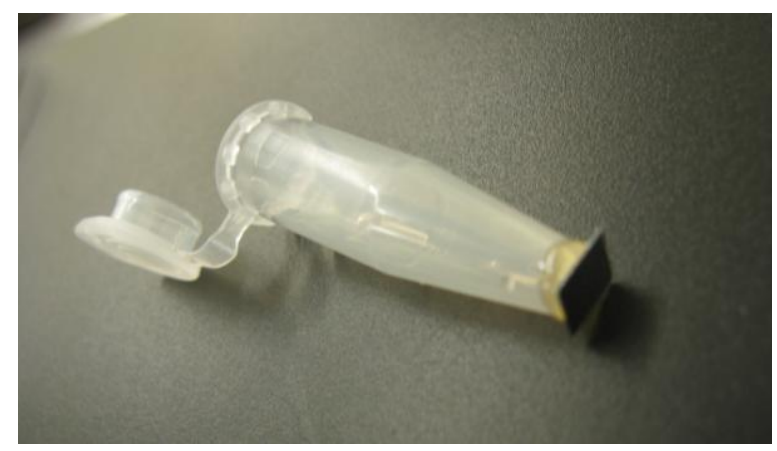

Figure 5. Silicon photonic chip with the array of sensors and the embedded microfluidic system incorporated in the bottom of a reaction tube.

\section{SETUP, EXPERIMENTS AND RESULTS}

\subsection{Setup and Experiments}

To perform the experiments, the device was fixed on a tiny chuck by means of vacuum. This chuck also has a connection to a pump, where pressure can be applied positively or negatively, pushing or sucking any gas of fluid applied in a specific area of this chuck. The reaction tube with the photonic chip integrated at its bottom was carefully aligned, so the 
perforations of the chip coincide with this area. Any fluid in contact with the chip will flow through the holes and be sucked or pushed up again by the pump. Figure 6a shows the device fixed on this chuck.

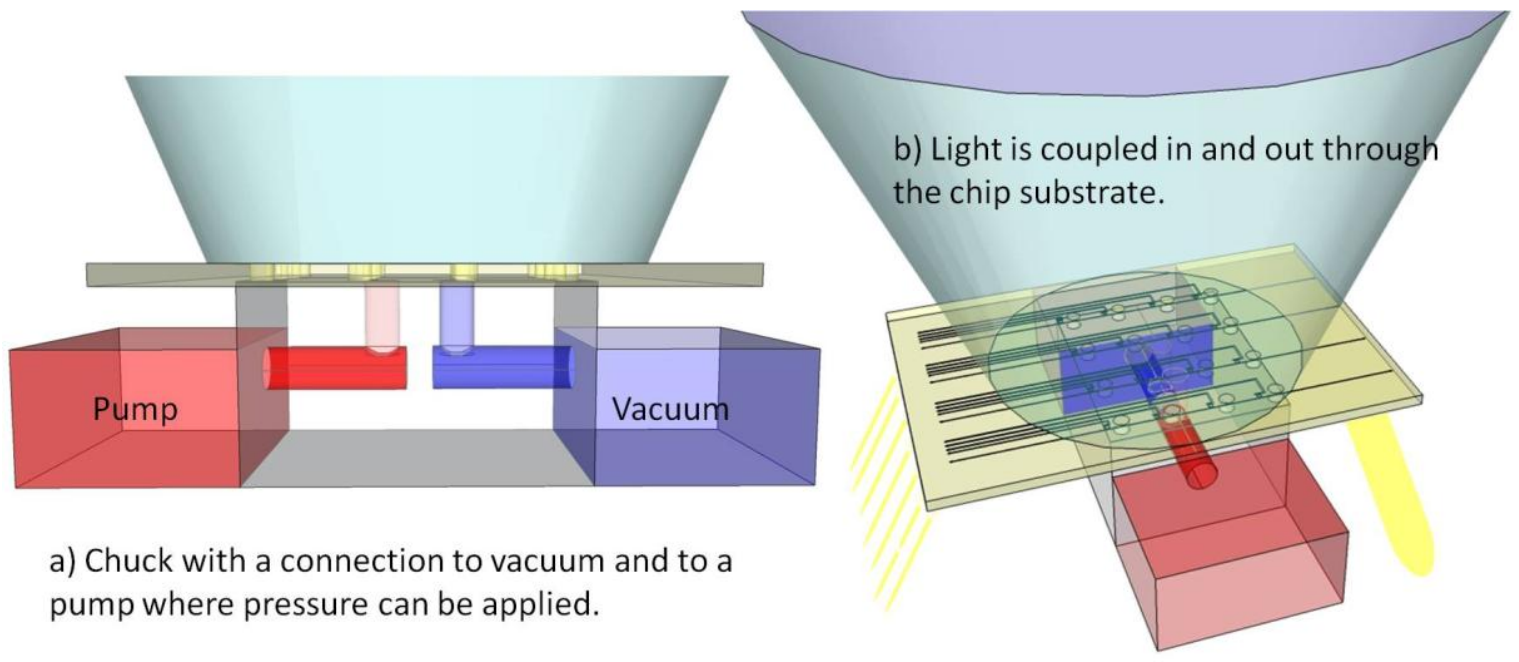

Figure 6. a) Device mounted on the chuck with a connection to vacuum and to a pump, where pressure can be applied in order to suck or push the fluid on the chip surface. b) The device is fixed to the chuck with vacuum. The measurements are performed from the bottom of the chip, i.e. light is coupled in and out through the chip substrate.

As described in Section 2.1, grating couplers were used to couple the light from a tunable laser into the chip and couple it out to be detected by an infrared camera. A new aspect with respect to our previous work ${ }^{2}$ is that now, we have the possibility of coupling light in and out from the bottom of the chip, i.e. through the $750-\mu \mathrm{m}$ thick silicon substrate. (Figure 6b) Silicon is considered practically transparent for the wavelength used $(1.55 \mu \mathrm{m})$. However, to reduce the scattering of the rough substrate surface and to facilitate the alignment of the laser beam and the detection of the light coupled out from the chip, a few simple processing steps were done in advance: the silicon substrate was thinned down to $300 \mu \mathrm{m}$ by chemical mechanical grinding and afterwards a chemical mechanical polishing step was performed in order to attain a smooth surface. Figure 7 shows an image from the IR camera where the chip is placed up-side down on a chuck that uses vacuum to keep the sensor fixed in place. The different photonic circuits of the SOI chip, the laser beam and even the vacuum holes of the chuck at the other side of the chip are clearly visible.

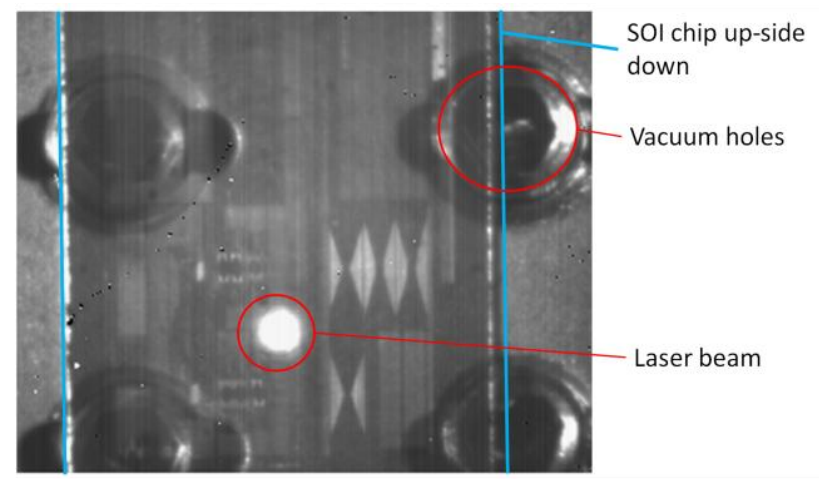

Figure 7. IR camera image. The chip is placed up-side down on a vacuum chuck. The laser beam and the vacuum holes of the chuck at the other side of the chip are clearly visible. The different photonic circuits on the SOI chip are distinguished as shadows and lighted areas in the picture.

As a proof-of-principle to show the capabilities of the combined device, we measured the different steps for an assay where the well-known high-affinity couple biotin-streptavidin was bound. The measurements were performed as follows: 
Small volumes of different solutions were manually pipetted in the tube, and they were sucked out through the perforations of the bottom of the tube while being measured. The experiment consists of three different steps.

1. The silanization of the surface: where a $2 \%$ solution of the aminosilane 3-Aminopropyl)triethoxysilane (APTES) was flowed preceded and followed by rinsing with ethanol.

2. The immobilization of biotin, where a solution $3 \mathrm{mg} / \mathrm{ml}$ of biotin in Phosphate Buffered Saline PBS was flowed preceded and followed by rinsing with PBS $\mathrm{pH} 7$.

3. The binding of streptavidin, where a solution of $0.1 \mathrm{mg} / \mathrm{ml}$ strepavidin in PBS was flowed preceded and followed by rinsing with PBS $\mathrm{pH} 7$.

The time to perform each of these steps was less than one hour.

\subsection{Results}

Figure 8 shows three different graphs corresponding to each one of the steps of the assay. They show the evolution in time of the resonance wavelength shift of the ring resonators during the measurements with different solutions. Each color corresponds to one sensor.
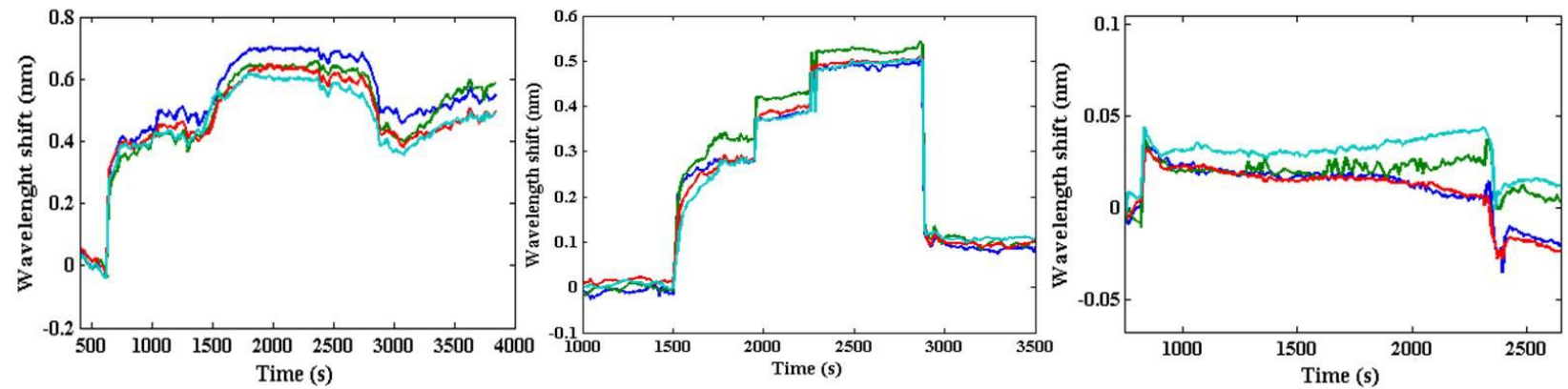

Figure 8. Three different steps of the bioassay were measured. From left to right. a) Silanization of the surface with APTES : ethanol-APTES- ethanol b) inmobilization of the biotin: PBS- biotin- PBS. c) binding of streptavidin to biotin: PBSstreptavidin- PBS

The association and disassociation of APTES in the sensor is easily quantifiable in Figure 6 Left. Figure 6 Center shows the binding of biotin after flowing $3 \mathrm{mg} / \mathrm{ml}$ solution of biotin in PBS and its disassociation when it is rinsed with PBS. A shift of $30 \mathrm{pm}$ is measured when streptavidin in flowed through the chip proving the binding of this to the biotin.

These measurements are preliminary results for the device just developed. Future work will imply the optimization of the device and the measurements.

\section{CONCLUSIONS}

We have presented the combination of a simple and compatible reaction tube platform, with label free nanophotonic biosensors and a special microfluidic system embedded in the same chip, showing that label-free nanophotonic ring resonators can also be used in a user-friendly platform like reaction tubes or well microtiter plates, conserving their excellent performance

\section{REFERENCES}

[1] Selvaraja S. K, Jaenen P, Bogaerts W, Van Thourhout D, Dumon P, and Baets R, "Fabrication of Photonic Wire and Crystal Circuits in Silicon-on-Insulator Using 193nm Optical Lithography," J. Lightwave Technol 27: 4076-4083(2009)

[2] De Vos K, Girones Molera J, Claes T, De Koninck Y, Popelka S, Schacht E., Baets R, Bienstman P.," Multiplexed antibody detection with an array of silicon-on-insulator microring resonators," IEEE Phot Journal, 1(4): 225-235 (2009) 
[3] Bogaerts W, Baets R, Dumon P, Wiaux V, Beckx S, Taillaert D, Luyssaert B, Van Campenhout J, Bienstman P, and Van Thourhout D, "Nanophotonic Waveguides in Silicon-on-Insulator Fabricated with CMOS Technology,”J. Lightw. Technol. 23, 1: 401-412 (2005) 